LBNL-62257

\title{
SELF-CONSISTENT 3D MODELING OF ELECTRON CLOUD DYNAMICS AND BEAM RESPONSE*
}

\author{
M. A. Furman, C. M. Celata, M. Kireeff-Covo, K. G. Sonnad, \\ J.-L. Vay ${ }^{\dagger}$, M. Venturini, LBNL, Berkeley, CA 94720-8211, USA \\ R. Cohen, A. Friedman, D. Grote, A. Molvik, LLNL, Livermore, CA 94550, USA \\ P. Stoltz, Tech-X Corp., Boulder, CO 80303, USA
}

\begin{abstract}
We present recent advances in the modeling of beamelectron-cloud dynamics, including surface effects such as secondary electron emission, gas desorption, etc, and volumetric effects such as ionization of residual gas and charge-exchange reactions. Simulations for the HCX facility with the code WARP/POSINST will be described and their validity demonstrated by benchmarks against measurements. The code models a wide range of physical processes and uses a number of novel techniques, including a large-timestep electron mover that smoothly interpolates between direct orbit calculation and guiding-center drift equations, and a new computational technique, based on a Lorentz transformation to a moving frame, that allows the cost of a fully 3D simulation to be reduced to that of a quasi-static approximation.
\end{abstract}

\section{INTRODUCTION}

The desire to increase the beam intensity in operational and upcoming accelerators leads to continuing concerns over the detrimental effects from electron clouds and gas pressure rise [1]. In addition, three-dimensional (3D) effects may become significant in future machines such as the ILC damping ring, which is dominated by wiggler magnets, and for the high-intensity accelerators envisioned for Heavy Ion Inertial Fusion (HIF) drivers and Warm-Dense Matter (WDM) studies. In the first case, the 3D effects arise from the intrinsically $3 \mathrm{D}$ field geometry of the wigglers, and in the second from the very long beam pulses which simultaneously encompass many lattice elements. To this end, we have undertaken the development of a new generation of computer simulation code in conjunction with detailed measurements for extensive code validation at a heavily diagnosed dedicated facility at LBNL, namely the High-Current Experiment (HCX) [2].

Our simulation tool, described in more detail below, has a fully self-consistent simulation (FSCS) capability in 3D, but can also be operated in simpler modes such as 2D build-up mode (BUM) and in quasi-static mode (QSM) (see below). By using the code in these simpler modes,

\footnotetext{
* Work supported by the U.S. DOE under Contracts DE-AC0205CH11231 and W-7405-Eng-48, by the US-LHC Accelerator Research Project (LARP), and by the FNAL Main Injector upgrade program. Paper TUXAB03, Proc. PAC07 (Albuquerque, NM, June 25-29, 2007), http://pac07.org/

† jlvay@lbl.gov
}

we have carried out successful code-to-code benchmarks against other codes, such as POSINST [3-6] and HEADTAIL $[7,8]$ in the context of high-energy particle accelerator models.

The FSCS 3D mode is, of course, the most complicated in terms of the simulation model and the most computationally costly. In part, the complexity is due to the large spread in time and length scales involved in the model. Recently we have developed a novel computational algorithm, applicable to relativistic beams, that significantly increases the computational speed of the 3D FSCS by a judicious choice of a moving frame of reference [9]. Preliminary results were presented recently [10-12].

\section{THE WARP-POSINST PACKAGE}

The simulation tool is based on a merge of the HIF code WARP [13] and the high-energy physics electron cloud build-up code POSINST [3-6], supplemented by additional modules for gas generation and ionization [10], as well as ion-induced electron emission from the Tech-X package TxPhysics [14]. The tool allows for multi-dimensional (2D or 3D) modeling of a beam in an accelerator lattice and its interaction with electron clouds generated from photon-induced, ion-induced or electron-induced emission at the vacuum chamber walls, or from ionization of background and desorbed gas. The generation and tracking of all species (beams particles, ions, electrons, gas molecules) is performed in a self-consistent manner (the electron, ion and gas distributions can also be prescribed -if needed-for special studies or convenience). The code runs in parallel and benefits from adaptive mesh refinement (AMR) [15], particle sub-cycling [16], and a new drift-Lorentz particle mover for tracking charged particles in magnetic fields using large time steps $[17,18]$. These advanced numerical techniques allow for significant speed-up in computing time (orders of magnitude) relative to brute-force integration techniques, allowing for self-consistent simulations of electron-cloud effects and beam dynamics, which were out of reach with previously available tools. Fig. 1 is a schematic illustration of the different functional modules in WARP-POSINST and their inter-relationships that are ultimately needed to reach FSCS [17-20].

Self-consistent simulation of electrons and beam particles requires simulation involving a broad range of time scales, ranging from the electron cyclotron period $\left(10^{-11}\right.$ 


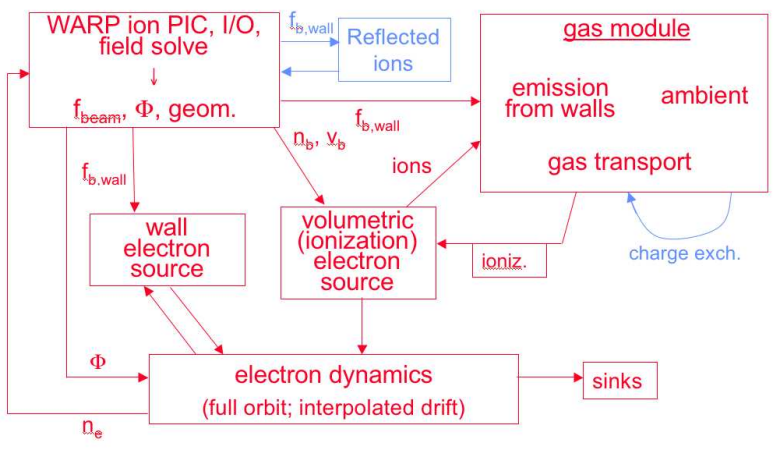

Figure 1: Sketch of the different functional modules in WARP-POSINST. At present, most modules are operational (red). The reflected ions and the charge exchange modules are still being developed (blue).

$\left.10^{-10} \mathrm{~s}\right)$ up to the beam transit time $\left(10^{-7}-10^{-5} \mathrm{~s}\right)$ through a series of lattice elements. The shortest electron cyclotron period is typically one to two orders of magnitude smaller than the next-smallest time scale. To deal with this large range of time scales in a unified manner, we have developed a mover for electrons that interpolates between full electron dynamics and drift kinetics $[17,18]$. This mover allows to integrate the motion of a charged particle in a magnetic field using time steps greater than the local cyclotron period, at the cost of a possible loss of information of the phase of the cyclotron motion. This drawback can be dealt with if necessary, but it is often inconsequential in practice.

Impact of energetic beam particles on the vacuum chamber surface can lead to desorption of neutral atoms or molecules. At high beam energies electronic sputtering is the dominant desorption mechanism. The model used by WARP for the energy and angular distribution of the desorbed neutrals is based on molecular dynamics calculations $[10,19]$.

The background gas, or the gas generated by wall desorption from beam particle impact, can be ionized (possibly fragmented) by the beam, gas ions or the electrons. Conversely, the interaction between the gas and the beam can lead to stripping, or capture of electrons by the beam particles. We track these events using a Monte Carlo scheme similar to the one described in [21]. For simplicity, we make the additional assumption (valid for current applications) that the gas reservoir is large enough, and the crosssections are small enough, that the depletion of gas due to its ionization can be neglected [10].

Although the code WARP-POSINST is fundamentally geared towards FSCS, and it allows to study in detail the beam and electron clouds for many turns, the 3D selfconsistent approach, even with the mesh refinement, subcycling and advanced particle pusher capabilities, must be supplemented by simplified descriptions for convenience and for benchmarking purposes. In BUM, the code forces the beam to be non-dynamical, i.e., the beam is represented

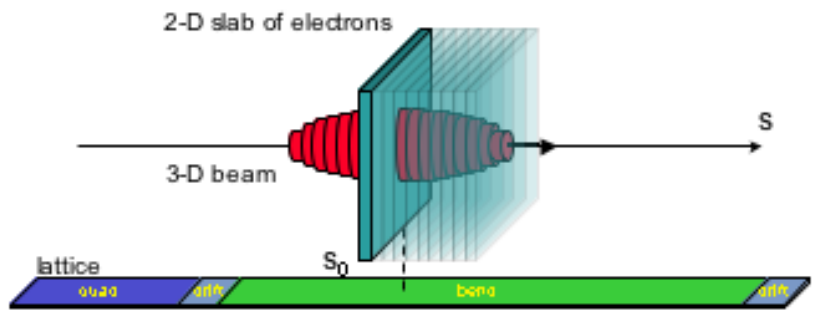

Figure 2: Cartoon explaining the QSM algorithm for the passage of a bunch through an ecloud station.

by a prescribed function of space and time, while the electrons are tracked fully dynamically in either $2 \mathrm{D}$ or $3 \mathrm{D}$, including primary and secondary emission. This mode of operation, is by definition, not self-consistent but it can provide valuable results in many cases. A benchmark of WARP in 2D BUM against POSINST for the case of an ILC dipole shows good agreement [22].

Another simplified mode of operation is the QSM, similar to what is used in the codes HEADTAIL $[7,8]$ or QUICKPIC [23,24]. In QSM the beam particles are tracked in 3D while the electrons are represented as twodimensional slices passing through the beam at one or more discrete points along the ring, called "ecloud stations" (see Fig. 2). The electron density at any given station is typically assumed to be uniform and cold just before the arrival of the bunch. After passing through a station, the beam particles are tracked through the lattice to the next station. For this, WARP allows either the use of a lattice map, or uses leap-frog integration through the various intervening lattice elements. In QSM, the beam particles and the electrons evolve under their mutual influence during the passage of the bunch through a station, but it is not fully self-consistent. This mode is much faster than FSCS and allows parametric studies of the thousands of turns that are required for the modeling of slow emittance growth, which is a concern for the LHC [25]. Fig. 3 shows a benchmark of WARP against HEADTAIL [7,8] for the case of 1 or 2 stations per turn for the propagation of a $20-\mathrm{GeV}$ proton beam with $10^{11}$ particles per bunch through a $5-\mathrm{km}$ ring with constant focusing [11] and a uniform electron cloud of density $10^{12} \mathrm{~m}^{-3}$.

\section{THE HIGH CURRENT EXPERIMENT}

The HCX [2], located at the Lawrence Berkeley National Laboratory, is a small ( $\sim 10 \mathrm{~m}$-long) heavy-ion linac consisting of an injector producing a singly-charged Potassium ion beam $(\mathrm{K}+)$ at $1 \mathrm{MeV}$ kinetic energy, followed by a transport lattice made of a matching section, a ten-quadrupole electrostatic section, and a four-quadrupole magnetic section. The flat top of the beam pulse reaches $180 \mathrm{~mA}$ and its duration is $4 \mu \mathrm{s}$.

The HCX is dedicated to the study of space-charge dominated beam transport and electron-cloud effects on the beam. Copious electrons are generated by slamming the 

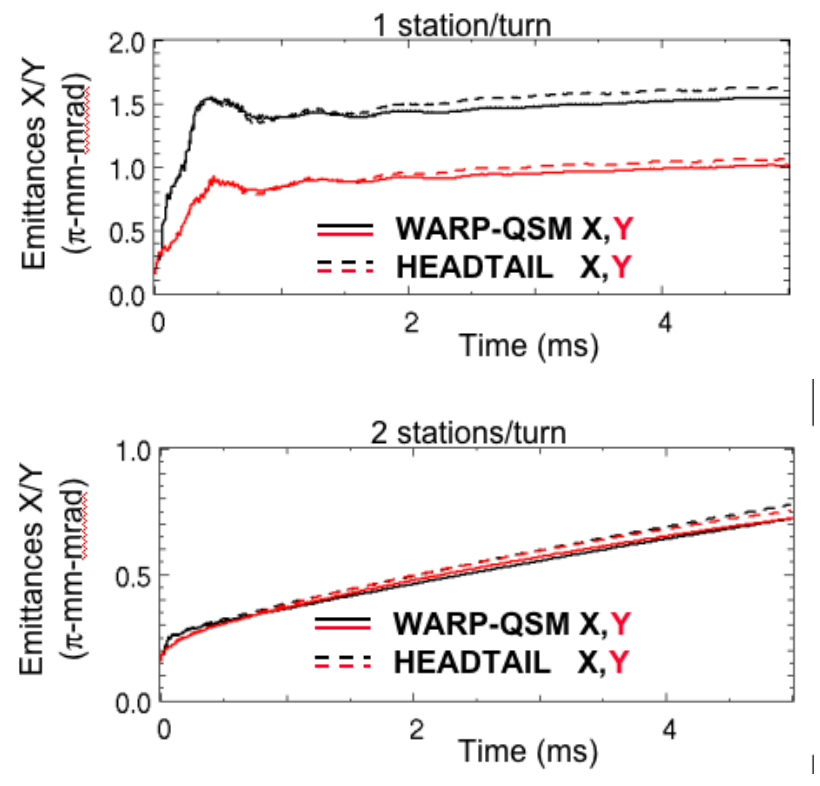

Figure 3: Comparison of results from WARP in QSM mode and HEADTAIL [7,8]. Shown are the RMS emittances vs. time for a $20-\mathrm{GeV}$ bunch of $10^{11}$ protons propagating through an electron cloud of density $10^{12} \mathrm{~m}^{-3}$. Top (bottom): one(two) ecloud stations per turn.

beam onto the end plate. We study electron effects in the magnetic section, which is heavily instrumented with diagnostic devices and suppressor electrodes dedicated to the various measurements of electron flux, electron energy spectrum, and gas analysis [26-32]. The primary electrons created at the end plate propagate upstream, entering only two quadrants of the fourth (last) magnet because of the sign of $\mathbf{E} \times \mathbf{B}$. The electron current measured by one of the clearing electrodes is compared with the simulation in Fig. 4, in the case where the suppressor ring electrode was left grounded to allow electrons to propagate upstream, and the three clearing electrodes were biased to $+9 \mathrm{kV}$. The simulation and experimental results show excellent agreement both on the magnitude and frequency $(\sim 10 \mathrm{MHz})$ of the observed oscillations. Simulation results reveal that these time-dependent oscillations are related to bunching of electrons drifting upstream in the fourth magnet. The nature of these oscillations has yet to be firmly identified. Although some possible explanations for this bunching have been excluded (electron-ion two-stream instability, for example), the topic is under active investigation.

\section{HIGH-ENERGY ACCELERATORS}

We have applied the WARP-POSINST code to the modeling of a train of bunches in one Large Hadron Collider (LHC) FODO cell in fully self-consistent mode. The magnetic fields in the FODO cell used in our simulation have nominal values for $7 \mathrm{TeV}$ beam energy, with geometry, dimensions and optics as specified in the LHC CDR [33].

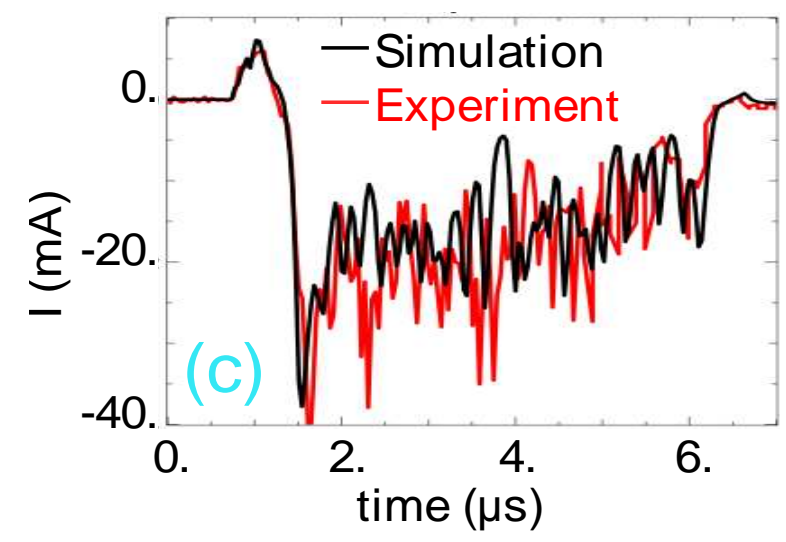

Figure 4: Electron current at clearing electrode (c). Red: recorded on HCX experiment; black: WARP-POSINST simulation.

However, for the purposes of these preliminary studies, we have used the following simplifications: (1) cell magnets other than dipoles and quadrupoles are not included (actually, replaced by drifts); and (2) magnetic edge fields are neglected. As for the bunch train, we represent it by a succession of identical bunches with nominal intensity, emittances, and spacing, but we use the following simplifications: (3) periodic boundary conditions in the longitudinal dimension, both for the beam and for the electrons (so that, effectively, the model represents a circular "storage ring" consisting of a single FODO cell); and (4) the energy spread is zero (all particles have nominal energy). A snapshot from the simulation of a train of five bunches is shown in Fig. 5 [12].

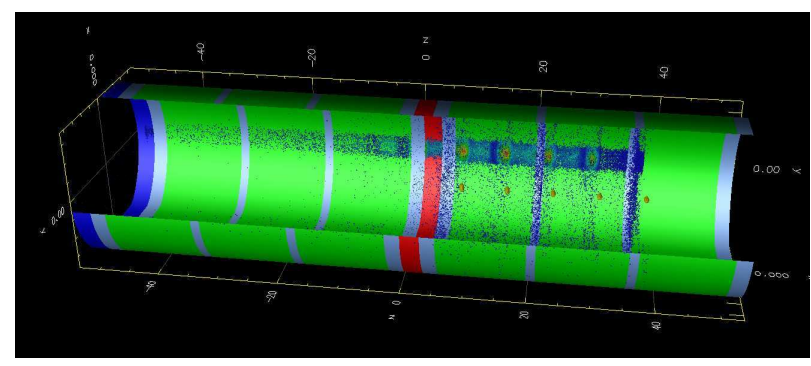

Figure 5: Snapshot from a 3D self-consistent simulation of five bunches propagating from left to right in one LHC arc FODO cell (green: dipoles; blue: focusing quad; red: defocusing quad; silver: drift). The electrons are generated by photo-emission at the rate of $1.27 \times 10^{-3}$ photoelectrons per proton per meter, preferentially at the outer edge of the chamber. Peak secondary emission yield is 2.0. Electrons are colored according to density (low: blue; high: red).

An application of WARP in QSM mode to an SPS-like machine model is shown in Fig. 3. An application the FNAL Main Injector proton ring [34] is discussed in [35]. An application to the LHC is shown in Fig. 6, which shows the simulated fractional emittance growth in a single turn as a function of the number of ecloud stations for a rather high 
ecloud density. It is evident that convergence is reached when the number of ecloud stations is several times the tune or, in other words, when the betatron wavelength is resolved.

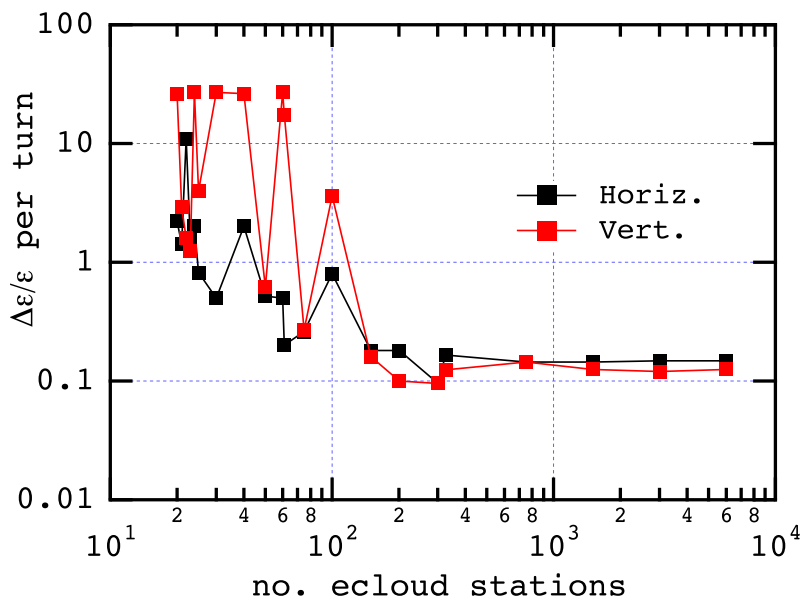

Figure 6: Simulated fractional emittance growth of the LHC beam over one turn as a function of the number of ecloud stations around the ring, assuming an electron density $n_{e}=10^{14} \mathrm{~m}^{-3}$. Beam conditions were $E=450 \mathrm{GeV}$, $N_{b}=1.1 \times 10^{11}$. The lattice was assumed to be constant focusing with tunes $\left(\nu_{x}, \nu_{y}\right)=(64.28,59.31)$.

\section{LORENTZ-BOOSTED FRAME}

Even with AMR, subcycling and the efficient driftLorentz mover, the FSCS of a beam through an electron cloud is a very expensive computational problem owing to the wide spread of the space and time scales involved. Such a problem arises in other areas of physics, e.g. the radiation from an electron bunch in a wiggler in an FEL. Such problems are called "multiscale" problems, and usually require massively parallel computations. Recently, one of us [9] observed that, if the beam is relativistic, the computational expense is substantially reduced by a judicious choice of a moving frame of reference. The relativistic contraction of length and dilatation of time affords a better matching of the time and length scales in the problem. The appropriate Lorentz factor of this boosted frame is somewhere between $\gamma=1$ (the Lab frame) and the $\gamma$ of the beam.

A simple example offers some intuitive understanding how this works: while the number of steps required to resolve, say, one cyclotron period of an electron is a Lorentz invariant, the key point is that, in a suitable boosted frame, the number of steps required to resolve the electron cyclotron period would also allow one to resolve the beam particle motion through one betatron wavelength due to the Lorentz contraction of the latter, while in the Lab frame one would need many more time steps to do so. Fig. 7 shows a comparison of the calculation of the RMS radius of a proton beam undergoing a hose instability as it traverses an electron cloud [9]. In this case, the brute-force computation in the Lab frame took more than 2 weeks of CPU time, while the in the boosted frame it took less than $30 \mathrm{~min}$, a speed-up factor of $10^{3}$, with essentially identical results.

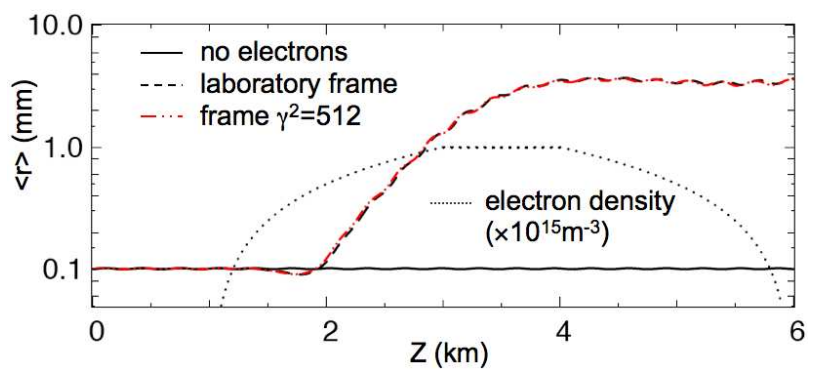

Figure 7: Evolution of a bunch of $10^{12}$ protons traversing an electron cloud. There is no vacuum chamber in this simulation. A magnetic field $B_{\theta}=$ const. $\times r$ provides focusing. The bunch traverses a total distance of $6 \mathrm{~km}$, of which the electron cloud occupies the last 5 $\mathrm{km}$ (dotted line). The electron density reaches a peak of $10^{15} \mathrm{~m}^{-3}$. The bunch and the boosted frame had Lorentz factors $\gamma=500$ and $\gamma=512^{1 / 2}$, respectively, relative to the Lab frame. Reprinted figure with permission from J.-L. Vay, Phys. Rev. Lett. 98, 130405 (2007). http://link.aps.org/abstract/PRL/v98/e130405. Copyright (2007) by the American Physical Society. Readers may view, browse, and/or download material for temporary copying purposes only, provided these uses are for noncommercial personal purposes. Except as provided by law, this material may not be further reproduced, distributed, transmitted, modified, adapted, performed, displayed, published, or sold in whole or part, without prior written permission from the American Physical Society.

The boosted frame technique makes the computational cost of a FSCS comparable of that of the QSM mode. It is of no benefit in the QSM approximation, nor in buildup simulations. The technique, however, brings up issues that remain to be addressed, for example: the curved trajectory of a beam through a bending magnet makes it not obvious how to choose the Lorentz boost (or, one might have to use general relativity to carry out a transformation to a non-inertial frame). The shift in the simultaneity of events requires a non-trivial translation of the particle coordinates in the boosted frame to the Lab frame; this translation would be required for full diagnostic purposes, for example. A pure magnetic field in the Lab frame shows up as a combined electric-magnetic field in the boosted frame, therefore the code must allow for particle tracking in an arbitrary electromagnetic field (which WARP does). Finally, the vacuum chamber in the boosted frame is a moving conductor, which requires an unconventional specification of the electric and magnetic boundary conditions for the field solver. Nevertheless, the large speed-up factor exhibited by of the Lorentz-boost technique in simple cases makes it highly desirable to solve these issues for more practical 
applications. This is an area of active research.

\section{CONCLUSION}

We have developed a three-dimensional self-consistent code suite which includes new numerical methods, allowing the modeling of accelerator configurations that were previously out of reach. Benchmarking against the HCX experiment has provided excellent agreement, and is being further pursued in order to fully validate the code and the embodied physical model. The code is also being applied to the self-consistent modeling of electron cloud effects in several high-energy storage rings such as the LHC, SPS, ILC damping ring and the FNAL Main Injector. Benchmarks of the code in QSM against HEADTAIL shows good agreement, as does a benchmark in 2D BUM against POSINST. A new algorithm, based on a Lorentz boost to a moving frame of reference, shows high promise of a significant reduction of the computational cost of a FSCS of a relativistic beam through an electron cloud and other similar relativistic multiscale problems.

\section{ACKNOWLEDGMENTS}

We are indebted to P. Seidl and J. Coleman for experimental assistance, and to NERSC for supercomputer support.

\section{REFERENCES}

[1] Proc. Intl. Wkshp. on Electron-Cloud Effects "ECLOUD07" (Daegu, S. Korea, April 9-12, 2007), http://chep.knu.ac.kr/ecloud07/

[2] L. R. Prost, P. A. Seidl, F. M. Bieniosek, C. M. Celata, A. Faltens, D. Baca, E. Henestroza, J. W. Kwan, M. Leitner, W. L. Waldron, R. Cohen, A. Friedman, D. Grote, S. M. Lund, A. W. Molvik, E. Morse, "High current transport experiment for heavy ion inertial fusion," PRST-AB 8 020101, (2005).

[3] M. A. Furman and G. R. Lambertson, "The electron-cloud instability in the arcs of the PEP-II positron ring," LBNL41123/CBP Note-246, PEP-II AP Note AP 97.27 (Nov. 25, 1997). Proc. Intl. Workshop on Multibunch Instabilities in Future Electron and Positron Accelerators "MBI-97" (KEK, 15-18 July 1997; Y. H. Chin, ed.), KEK Proceedings 97-17, Dec. 1997, p. 170.

[4] M. A. Furman and M. T. F. Pivi, "Probabilistic model for the simulation of secondary electron emission," LBNL49771/CBP Note-415 (Nov. 6, 2002). PRST-AB 5124404 (2003), http://prst-ab.aps.org/pdf/PRSTAB/v5/i12/e124404.

[5] M. A. Furman and M. T. F. Pivi, "Simulation of secondary electron emission based on a phenomenological probabilistic model," LBNL-52807/SLAC-PUB-9912 (June 2, 2003).

[6] M. A. Furman, "The electron-cloud effect in the arcs of the LHC," LBNL-41482/CBP Note 247/LHC Project Report 180 (May 20, 1998).

[7] http://wwwslap.cern.ch/collective/electroncloud/Programs/Headtail/headtail.html
[8] G. Rumolo and F. Zimmermann, "Practical User Guide for HEADTAIL,” CERN-SL-Note-2002-036 AP (2002).

[9] J.-L. Vay, "On the non-invariance of space and time scale ranges under Lorentz transformation, and its implications for the study of relativistic interactions," Phys. Rev. Lett. 98, 130405 (2007).

[10] J.-L. Vay, M. A. Furman, R. H. Cohen, A. Friedman and D. P. Grote, "Initial Self-Consistent 3D Electron-Cloud Simulations of the LHC Beam with the Code WARP+POSINST," Proc. PAC05 (Knoxville, TN, 16-20 May 2005), paper FPAP016.

[11] J.-L. Vay, A. Friedman, D. P. Grote, "Self-Consistent Simulations of High-Intensity Beams and E-Clouds with WARPPOSINST," Proc. ICAP06 (Chamonix, Oct. 2-6, 2006), paper WEA3MP02.

[12] J.-L. Vay, M. A. Furman, P. A. Seidl, R. H. Cohen, A. Friedman, D. P. Grote, M. Kireeff Covo, A. W. Molvik, P. H. Stoltz, S. Veitzer and J. P. Verboncoeur, "Self-consistent simulations of heavy-ion beams interacting with electron clouds," Proc. 16th Intl. Symp. on Heavy-Ion Inertial Fusion "HIF06" (Saint-Malo, France, 9-14 July, 2006). Published: NIMPR A 577 (2007), 65.

[13] D. P. Grote, A. Friedman, J.-L. Vay. I. Haber, AIP Conf. Proc. 74955 (2005).

[14] P. H. Stoltz, J. P. Verboncoeur, R. H. Cohen, A. W. Molvik, J.-L. Vay, S. A. Veitzer, "Modeling ion-induced electrons in the High Current Experiment," Phys. of Plasmas 13, 056702 (2006).

[15] J.-L. Vay, P. Colella, J. W. Kwan, P. McCorquodale, D. B. Serafini, A. Friedman, D. P. Grote, G. Westenskow, J.-C. Adam, A. Héron, I. Haber, "Application of adaptive mesh refinement to particle-in-cell simulations of plasmas and beams," Phys. of Plasmas 11, 2928 (2004).

[16] J.-C. Adam, A. Gourdin Serveniere and A. B. Langdon, "Electron sub-cycling in particle simulation of plasma," J. Comp. Phys. 47, 229 (1982).

[17] R. H. Cohen, A. Friedman, D. P. Grote, J.-L. Vay, "Largetimestep mover for particle simulations of arbitrarily magnetized species," Proc. 16th International Symposium on Heavy Ion Inertial Fusion, HIF2006 (Saint-Malo, France, July 9-14, 2006). NIMPR A 577, 52 (2007).

[18] R. H. Cohen, A. Friedman, M. Kireeff Covo, S. M. Lund, A. W. Molvik, F. M. Bieniosek, P. A. Seidl, and J.-L. Vay, P. Stoltz and S. Veitzer, "Simulating electron clouds in heavyion accelerators," Physics of Plasmas 12, 056708 (2005)

[19] A. W. Molvik, Michel Kireeff Covo, F. M. Bieniosek, L. Prost, P. A. Seidl, D. Baca, A. Coorey, A. Sakumi, "Gas desorption and electron emission from $1 \mathrm{MeV}$ potassium ion bombardment of stainless steel," PRST-AB 7, 093202 (2004).

[20] P. H. Stoltz, M. A. Furman, J.-L. Vay, A. W. Molvik and R. H. Cohen, "Numerical Simulation of the Generation of Secondary Electrons in the High Current Experiment," PRSTAB 6, 054701 (2003).

[21] C. K. Birdsall, "Particle-in-cell charged-particle simulations, plus Monte Carlo collisions with neutral atoms, PICMCC,” IEEE Trans. Plasma Sci. 19(2), 65 (1991). 
[22] C. M. Celata, M. Furman, J.-L. Vay, M. Venturini, D. Grote, "Particle-in-Cell Calculations of the Electron Cloud in the ILC Positron Damping Ring Wigglers," Proc. PAC07, paper TUPMN108.

[23] G. Rumolo, A. Z. Ghalam, T. Katsouleas, C. K. Huang, V. K. Decyk, C. Ren, W. B. Mori, F. Zimmermann, F. Ruggiero, "Electron cloud effects on beam evolution in a circular accelerator," PRST-AB 6081002 (2003).

[24] A. Z. Ghalam, T. Katsouleas, V. K. Decyk, C. K. Huang, W. B. Mori, G. Rumolo, E. Benedetto, F. Zimmermann, "3D Parallel Simulation Model of Continuous Beam Electron Cloud Interactions," Proc. PAC05 (Knoxville, TN, 1620 May 2005), paper ROPB007.

[25] E. Benedetto, D. Schulte, F. Zimmermann, G. Rumolo, "Simulation study of electron cloud induced instabilities and emittance growth for the CERN Large Hadron Collider proton beam," PRST-AB 8, 124402 (2005).

[26] A. W. Molvik, M. Kireeff Covo, A. Friedman, R. Cohen, S. M. Lund, J. J. Barnard, F. Bieniosek, P. Seidl, D. Baca, J.-L. Vay, C. M. Celata, W. L. Waldron, J. L. Vujic, "Experiments Studying Desorbed Gas and Electron Clouds in Ion Accelerators," Proc. PAC05, paper ROPB002

[27] A. Molvik, R. Cohen, A. Friedman, M. Kireeff Covo, F. Bieniosek, P. Seidl, and J.-L. Vay, "New experimental measurements of electron clouds in ion beams with large tune depression," Proc. 39th ICFA Advanced Beam Dynamics Workshop, High Intensity High Brightness Hadron Beams "HB2006" (Tsukuba, Japan, May 29 - June 02, 2006), paper THAW02.

[28] A. W. Molvik, M. Kireeff Covo, R. H. Cohen, A. Friedman, W. M. Sharp, D. Baca, F. M. Bieniosek, C. Leister, P. A. Seidl and J.-L. Vay, "Quantitative electron and gas cloud experiments," Proc. 16th Intl. Symp. Heavy Ion Inertial Fusion "HIF2006" (Saint-Malo, France, July 9-14, 2006). NIMPR A 577, 45 (2007).

[29] A. W. Molvik, H. Kollmus, E. Mahner, M. Kireeff Covo, M. C. Bellachioma, M. Bender, F. M. Bieniosek, E. Hedlund, A. Krämer, J. Kwan, O. B. Malyshev, L. Prost, P. A. Seidl, G. Westenskow, L. Westerberg, "Electronic desorption of gas from metals," Phys. Rev. Lett. 98, 064801 (2007).

[30] A. W. Molvik, M. Kireeff Covo, R. Cohen, A. Friedman, S. M. Lund, W. Sharp, J.-L. Vay, D. Baca, F. Bieniosek, C. Leister, and P. Seidl, "Quantitative experiments with electrons in a positively charged beam," Phys. of Plasmas 14, 056701 (2007)

[31] M. Kireeff Covo, A. W. Molvik, A. Friedman, J. J Barnard, P. A. Seidl, G. Logan, D. Baca and J. L. Vujic, "Beam Interaction Measurements with a Retarding Field Analyzer in a High-Current High-Vacuum Positively-Charged Particle Accelerator," Proc. 16th Intl. Symp. on Heavy Ion Inertial Fusion "HIF2006," (Saint-Malo, France, July 9-14, 2006). NIMPR A 577, 139 (2007).
[32] M. Kireeff Covo, A. W. Molvik, A. Friedman, J.-L. Vay, P. A. Seidl, G. Logan, D. Baca and J. L. Vujic, "Absolute Measurement of Electron Cloud Density in a Positively-Charged Particle Beam,” Phys. Rev. Lett. 97, 54801 (2006).

[33] The Large Hadron Collider: Conceptual Design, CERN/AC/95-05 (LHC), Oct. 1995.

[34] Proton Driver Study. II. (Part 1, ch. 13), FERMILAB-TM2169 (G. W. Foster, W. Chou and E. Malamud, eds.), May 2002.

[35] K. G. Sonnad, C. M. Celata, M. Furman, D. Grote, J.-L. Vay, M. Venturini, "Simulations of Electron Cloud Effects on the Beam Dynamics for the FNAL Main Injector Upgrade," Proc. PAC07, paper FRPMS028.

\section{DISCLAIMER}

This document was prepared as an account of work sponsored by the United States Government. While this document is believed to contain correct information, neither the United States Government nor any agency thereof, nor The Regents of the University of California, nor any of their employees, makes any warranty, express or implied, or assumes any legal responsibility for the accuracy, completeness, or usefulness of any information, apparatus, product, or process disclosed, or represents that its use would not infringe privately owned rights. Reference herein to any specific commercial product, process, or service by its trade name, trademark, manufacturer, or otherwise, does not necessarily constitute or imply its endorsement, recommendation, or favoring by the United States Government or any agency thereof, or The Regents of the University of California. The views and opinions of authors expressed herein do not necessarily state or reflect those of the United States Government or any agency thereof, or The Regents of the University of California.

Ernest Orlando Lawrence Berkeley National Laboratory is an equal opportunity employer. 